\title{
New targets bring hope in squamous cell lung cancer: neurotrophic tyrosine kinase gene fusions
}

\author{
Christian Rolfo ${ }^{1}$ and Luis Raez ${ }^{2}$
}

Neurotrophic tyrosine kinase genes encode for the Trk-family proteins TrkA, TrkB, and TrkC, which have an important role in the development of the nervous system; however, they have been identified as oncogenic fusions in solid tumors (NTK-1, NTRK-2, and NTRK-3) and are associated with poor survival in lung cancer. These three new fusions can be detected by fluorescent in situ hybridization or next-generation sequencing in less than $5 \%$ of the lung tumors. There are several ongoing clinical trials of NTRK oncogenes in lung cancer and other tumors. The agents entrectinib (RXDX-101), a multi-kinase small molecule inhibitor that selectively inhibits NTRK1, NTRK2, and NTRK3, ROS1 and ALK, and LOXO-101, an ATP-competitive pan-NTRK inhibitor, have shown responses in patients with lung cancer with an acceptable toxicity profile. Although these oncogenic fusions are not very prevalent, the high prevalence of lung cancer makes these findings very relevant and suggests the feasibility of these oncogenes as targets in lung cancer. New data from Ozono and collaborators presented in this issue suggest that BDNF/TrkB signal promotes proliferating migratory and invasive phenotypes and cellular plasticity in squamous cell carcinoma (SCC) of the lung but that it also represents a druggable target that may bring hope to squamous lung cancer patients.

Laboratory Investigation (2017) 97, 1268-1270; doi:10.1038/labinvest.2017.91

Phase I-Early Clinica Trials Unit, Oncology Department, Antwerp University Hospital \& Center for Oncology Research (CORE) Antwerp University, Antwerp, Belgium and

${ }^{2}$ Thoracic Oncology Program, Memorial Cancer Institute, Memorial Health Care System Oncology, Pembroke Pines, FL, USA Correspondence: E-mail: christian.rolfo@ uza.be

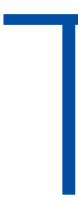
he therapeutic landscape of lung cancer has been changing dramatically since the introduction of therapuetic targets such as epidermal growth factor receptor (EGFR) and anaplastic lymphoma kinase (ALK) rearrangements. New, unusual suspects, including BRAF, RET, ROS1, among others are bringing new hope to patients and caregivers. One of the new kids on the block is neurotrophic tyrosine kinase (NTRK). NTRK genes encode for the Trk-family proteins: TrkA, TrkB, and TrkC (encoded by NTRK1, NTTRK2, and NTRK3). The NTRK family plays a role in the development of the nervous system with the modulation of growth, proliferation, repair, maintenance and apoptosis. ${ }^{1,2}$ However, NTRK fusions are also present in solid tumors as oncogenic fusions responsible for growth of cancer cells, proliferation, and survival; the presence of these three oncogenes is associate with poor survival in lung cancers. ${ }^{3,4}$ NTRK1 oncogenic fusions have been detected by fluorescent in situ hybridization (FISH) or next generation sequencing in about $3 \%$ of NSCLC, while NTRK2 and NTRK3 fusions in about 1\% of NSCLC of all types. ${ }^{3,5}$ Several therapeutic agents are currently in development for these oncogenes; one of them is entrectinib (RXDX-101) $)^{6,7}$ which is a multi-kinase small molecule inhibitor that selectively inhibits NTRK1, NTRK2, and NTRK3, ROS1 and ALK, as shown in vitro and in vivo in several tumor models. In mice xenografts of colorectal cancer cells with the TrkA fusion TPM3-NTRK1, treatment with entrectinib caused tumor regression. ${ }^{8}$ Other TRKA inhibitors such as CEP-701 and ARRY-470 were also successful against lung cancer cells expressing NTRK1 fusions. ${ }^{3}$ Another important agent is LOXO-101, an ATP-competitive pan-NTRK inhibitor that has shown antitumor activity against lung adenocarcinomas. ${ }^{9}$ AZD7451 was tested on colorectal cancer cell lines harboring tropomyosin (TPM)/NTRK1 fusion (KM12 cell line) and the H460 (an LCNEC cell line exhibiting NTRK2 expression). Proliferation was inhibited in KM12 and H460. An analysis of the H460 cells demonstrated decreased levels of phosphorylation 
of NTRK2 after treatment with AZD7451. ${ }^{10}$ These and other preclinical studies in colorectal cell lines suggested a role for targeting NRTK-dependent cancer cell populations because these are oncogenic derivers of the cancer tumors.

There are several clinical trials ongoing for NTRK oncogenes, for example, the agent entrectinib first showed activity in colorectal cancer harboring a TPM3-NTRK1 fusions. ${ }^{8}$ The phase I studies for ALKA-372-001 and STARTRK-1 were dose-escalation trials of entrectinib for patients with advanced or metastatic solid tumors harboring TrkA, ROS1, or ALK molecular alterations. The results of both trials with 67 patients enrolled ( 38 were NSCLC), were presented at the 2015 ASCO. ${ }^{11,12}$ Among 11 patients received the recommended phase 2 dose of entrectinib 10 responses were reported including 3 of 3 patients harboring NTRK translocations obtained a partial response. In another publication, Farago et al ${ }^{13}$ screened 1378 cases of NSCLC using PCR and confirmed by FISH the presence of NTRK1 gene rearrangements. Two patients $(0.1 \%)$ were found to have NTRK1 gene rearrangements: one was a TPM3-NTRK1 rearrangement, and in the second case, a fusion transcript containing sequence from SQSTM1 (sequestosome 1, which has previously been described as a fusion partner with ALK in NSCLC) and NTRK1 was observed. The patient presenting the SQSTM1/NTRK1 fusion participated in a phase 1 clinical trial with entrectinib and he achieved a partial response. There is an ongoing trial called STARTRK-2 (NCT02568267) which is a phase 2 basket study evaluating entrectinib for the treatment of solid tumors harboring NTRK1/2/ 3, ROS1, or ALK gene rearrangements currently recruiting patients in the US.

LOXO-101 is an ATP-competitive pan-Trk inhibitor, which has demonstrated encouraging activity on NTRK pre-clinical studies. A phase 1 trial (NCT02122913) is currently recruiting patients. ${ }^{14}$ The study has enrolled six patients with NTRK fusions, including one NSCLC (TPRNTRK1) two mammary analog secretory carcinoma (MASC) (ETV6-NTRK3), one thyroid carcinoma (ETV6-NTRK3), one GIST (ETV6NTRK3), and one soft tissue sarcoma (STS) (LMNA-NTRK1). All three patients evaluable for efficacy (STS, MASC, and GIST) reported partial responses to LOXO-101. ${ }^{15}$ Doebele et al published a case report reporting a PR and symptom improvement in a patient with a metastatic STS harboring the LMNA-NTRK1 gene fusion, participating in the LOXO-101 phase 1 trial. $^{9} \mathrm{~A}$ phase 2 basket study of LOXO-101 in patients NTRK fusion positive solid tumors, including CNS tumors is ongoing (NCT02576431). New drugs are currently under investigation to target tumors harboring activating NTRK molecular alterations in early phase I studies including patients with advanced solid tumors like the multi-target inhibitors XL-184, MGCD516, and DCC-2701 that are under evaluation in advanced cancers positive for different molecular alterations, including also NTRK (NCT02219711, NCT02228811). Among these, DCC-2701 (Altiratinib), a MET, TIE2, VEGFR2, and TRK kinase inhibitor currently in phase 1 clinical development has recently received orphan drug designation for glioblastoma by the US Food and Drug Administration (FDA). AZD7451 is a tyrosine kinase inhibitor that selectively targets NTRK1, NTRK2, and NTRK3 kinases, being considered then a pan NTRK kinase inhibitor which has shown promising pre-clinical activity in NTRK1 fusion positive lung cancer celllines. ${ }^{10}$ Finally, there is the TrkA/ALK inhibitor TSR-011 (NCT02048488), the pan-Trk/ROS1 inhibitor DS6051b (NCT02279433), and the panTrk inhibitor PLX-7486 (NCT01804530).

The study by Ozono et al ${ }^{16}$ in this issue of Laboratory Investigation hypothesizes that BDNF/ TrkB signal promotes proliferating migratory and invasive phenotypes and cellular plasticity in squamous cell carcinoma (SCC) of the lung. The investigators conclude that the $\mathrm{BDNF} / \mathrm{TrkB}$ signaling pathway contributes to the aggressive behavior of lung SCC by promoting cell invasion and proliferation, and suggest that this pathway may be a new therapeutic target for lung SCC. We agreed with the authors' conclusions. This is the first study to demonstrate, using a Trk inhibitor, that the inhibition of TrkB suppresses the migration, invasion and proliferation activities of lung SCC specifically. There are previous studies of Trk in colon cancer and lung cancer, but this paper is devoted exclusively to SCC, a type of cancer that is in urgently in need of new targets because most of the common actionable genes for lung cancer (EGFR, ALK, ROS-1, RET) are generally found in adenocarcinomas of the lung. These results may pave the way for a target therapy for lung SCC.

We know that NTRK fusions are not very common ( $<5 \%$ of the lung cancers) and that might tamper some enthusiasm, however due to the fact that lung cancers are very prevalent and numerous, even this number of patients represent a very significant group of patients in need of therapy in the US and worldwide. ${ }^{17}$ 


\section{DISCLOSURE/CONFLICT OF INTEREST}

LR has research support from LOXO oncology. The contents are solely the responsibility of the author and any opinions expressed herein are not necessarily the views of the editors, the United States and Canadian Academy of Pathology, or Springer Nature.

1. Brodeur GM, Minturn JE, Ho R, et al. Trk receptor expression and inhibition in neuroblastomas. Clin Cancer Res 2009;15:3244-3250.

2. Kaplan DR, Martin-Zanca D, Parada LF. Tyrosine phosphorylation and tyrosine kinase activity of the trk protooncogene product induced by NGF. Nature 1991;350: 158-160.

3. Vaishnavi A, Cappelletti M, Le AT, et al. Oncogenic and drug-sensitive NTRK1 rearrangements in lung cancer. Nat Med 2013;19:1469-1472.

4. Okamura K, Harada T, Wang S, et al. Expression of TrkB and $\mathrm{BDNF}$ is associated with poor prognosis in non-small cell lung cancer. Lung Cancer 2012;78:100-106.

5. Harada $T$, Yatabe $Y$, Takeshita $M$, et al. Role and relevance of TrkB mutations and expression in non-small cell lung cancer. Clin Cancer Res 2011;17:2638-2645.

6. Rolfo C, Ruiz R, Giovannetti E, et al. Entrectinib: a potent new TRK, ROS1, and ALK inhibitor. Expert Opin Investig Drugs 2015;24:1493-1500.

7. Passiglia F, Caparica R, Giovannetti E, et al. The potential of neurotrophic tyrosine kinase (NTRK) inhibitors for treating lung cancer. Expert Opin Investig Drugs 2016;25: 385-392.

8. Anderson D, Ciomei M, Banfi $P$ et al. Inhibition of Trkdriven tumors by the pan-Trk inhibitor RXDX-101. Eur J Cancer 2014;50:101.
9. Doebele RC, Davis LE, Vaishnavi A, et al. An oncogenic NTRK fusion in a patient with soft-tissue sarcoma with response to the tropomyosin-related kinase inhibitor LOXO-101. Cancer Discov 2015;5:1049-1057.

10. Tatematsu T, Sasaki H, Shimizu S, et al. Investigation of neurotrophic tyrosine kinase receptor 1 fusions and neurotrophic tyrosine kinase receptor family expression in non-small-cell lung cancer and sensitivity to AZD7451 in vitro. Mol Clin Oncol 2014;2:725-730.

11. De Braud FG, Niger M, Damian S et al. Alka-372-001: firstin-human, phase I study of entrectinib - an oral pan-trk, ROS1, and ALK inhibitor - in patients with advanced solid tumors with relevant molecular alterations. J Clin Oncol 2015;33:2517-2517.

12. Patel MR, Bauer TM, Liu SV et al. STARTRK-1: phase 1/2a study of entrectinib, an oral Pan-Trk, ROS1, and ALK inhibitor, in patients with advanced solid tumors with relevant molecular alterations. J Clin Oncol 2015;33:2596-2596.

13. Farago $A F$, Le LP, Zheng $Z$, et al. Durable clinical response to entrectinib in NTRK1-rearranged non-small cell lung cancer. J Thorac Oncol 2015;10:1670-1674.

14. Burris HA, Brose MS, Shaw AT et al. A first-in-human study of LOXO-101, a highly selective inhibitor of the tropomyosin receptor kinase (TRK) family. J Clin Oncol 2015;33:TPS2624.

15. TRK Inhibitor Shows Early Promise. Cancer Discov 2016; 6 : OF4.

16. Ozono $\mathrm{K}$, Ohishi $\mathrm{Y}$, Onishi $\mathrm{H}$, et al. Brain-derived neurotrophic factor/tropomyosin-related kinase B signaling pathway contributes to the aggressive behavior of lung squamous cell carcinoma. Lab Invest 201710.1038/ labinvest.2017.45.

17. Amantu A, Sartore Bianchi A, Siena S. NTRK gene fusions as novel targets of cancer therapy across multiple tumor types. ESMO Open 2016:1 e000023.ecollection2016. 